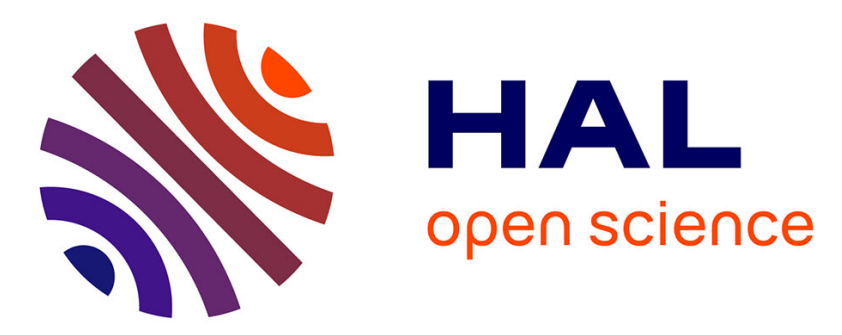

\title{
Encapsulation of a cationic antimicrobial peptide into self-assembled polyion complex nano-objects enhances its antitumor properties
}

\author{
Mina Răileanu, Barbara Lonetti, Charles-Louis Serpentini, Dominique \\ Goudounèche, Laure Gibot, Mihaela Bacalum
}

\section{To cite this version:}

Mina Răileanu, Barbara Lonetti, Charles-Louis Serpentini, Dominique Goudounèche, Laure Gibot, et al.. Encapsulation of a cationic antimicrobial peptide into self-assembled polyion complex nanoobjects enhances its antitumor properties. Journal of Molecular Structure, 2022, 1249, pp.131482. 10.1016/j.molstruc.2021.131482 . hal-03343639

\section{HAL Id: hal-03343639 \\ https://hal.science/hal-03343639}

Submitted on 14 Sep 2021

HAL is a multi-disciplinary open access archive for the deposit and dissemination of scientific research documents, whether they are published or not. The documents may come from teaching and research institutions in France or abroad, or from public or private research centers.
L'archive ouverte pluridisciplinaire HAL, est destinée au dépôt et à la diffusion de documents scientifiques de niveau recherche, publiés ou non, émanant des établissements d'enseignement et de recherche français ou étrangers, des laboratoires publics ou privés. 


\title{
Encapsulation of a cationic antimicrobial peptide into self-assembled polyion complex nano-objects enhances its antitumor properties
}

Mina Răileanu ${ }^{\mathrm{a}, \mathrm{b}}$, Barbara Lonetti ${ }^{\mathrm{c}}$, Charles-Louis Serpentini ${ }^{\mathrm{c}}$, Dominique Goudounèche ${ }^{\mathrm{d}}$, Laure Gibot $^{\text {c* }}$, Mihaela Bacalum ${ }^{\text {a* }}$

a Department of Life and Environmental Physics, "Horia Hulubei" National Institute for Physics and Nuclear Engineering, 30 Reactorului, Bucharest-Măgurele, Romania

${ }^{\mathrm{b}}$ Department of Electricity, Solid-State Physics and Biophysics, Faculty of Physics, University of Bucharest, 405 Atomistilor Street, Bucharest-Magurele, 077125, Romania;

${ }^{\mathrm{c}}$ Laboratoire des IMRCP, Université de Toulouse, CNRS UMR 5623, Université Toulouse III Paul Sabatier, France

${ }^{\mathrm{d}}$ Centre de Microscopie Électronique Appliquée à la Biologie, CMEAB, 133 route de Narbonne, 31062 Toulouse, France

* Corresponding authors: Dr. Mihaela Bacalum, email: bmihaela@nipne.ro, Dr. Laure Gibot, email: gibot@chimie.ups-tlse.fr

\begin{abstract}
Antimicrobial peptides, a large class of molecules synthetized by various organisms as an innate defense against pathogens are more and more used for their anticancer properties as well. In order to overcome some of their limitations and to enhance their therapeutic efficiency, the use of delivery systems was taken into consideration. In this study we describe an original delivery system for antimicrobial peptides based on its physico-chemical properties, namely the selfassembled polyion complexes (PIC) based on electrostatic interactions of cationic antimicrobial peptide P6 with negatively charged double hydrophilic block copolymers, the poly(ethylene oxide)-poly(acrylic acid) (PEO-PAA) in our study. The drug delivery system was tested on 3D human tumor HCT-116 spheroids. The spheroid evolution and cell viability were monitored at 24 and $48 \mathrm{~h}$ after the treatment was applied. Our study demonstrates for the first time the feasibility of forming a polyion complex PIC with an antimicrobial peptide and that this self-assembled organisation provides added value in terms of anti-tumour therapeutic efficacy compared to the free form of the antimicrobial peptide.
\end{abstract}

Keywords: micelle, poly(ethylene oxide)-poly(acrylic acid) (PEO-PAA), polymers, polyionic complex (PIC), self-assembly, spheroid 


\section{Introduction}

Despite an intense research, cancer treatment still remains a global problem for a number of cancer types: colorectal, lung, prostate, breast, etc. [1]. Although the use of conventional chemotherapy is generally effective, it is also responsible for the occurrence of toxic side effects in normal cells and tissues, exhibiting long-term negative consequences such as induction of secondary malignancies [2], which has a negative impact on patients' quality of life. In addition, the mechanisms underlying the carcinogenesis process and the mechanisms that induce the resistance of cancer cells to chemotherapy treatments are not yet fully understood, limiting the development of new drugs with increased therapeutic efficacy. The development of new anticancer therapeutic strategies that have both low toxicity, but no chemoresistance would be an important step forward in the fight against cancer. Thus, it has become imperative to find new agents who, alone or through delivery systems, can overcome these disadvantages. A promising class is antimicrobial peptides (AMPs).

AMPs are part of the innate defense mechanisms of many eukaryotic organisms, such as plants, insects, mammals, including humans and have been shown to be particularly effective in the fight against pathogens (bacteria, fungi, viruses) [3, 4]. To date, a wide variety of natural AMPs have been identified and characterized, which has led to the development of an even greater number of synthetic AMPs. Most of these AMPs are small peptides (less than 50 amino acids) and cationic, with a net charge on the molecule between +2 and +7 at physiological $\mathrm{pH}[5,6]$. Given that their structure plays a major role in their activity, the presence of specific amino acids can modulate their effectiveness. Thus, recent studies have shown that antimicrobial peptides rich in tryptophan and arginine have an increased potential against bacteria, fungi, but also against cancer cells [7, 8].

Despite these advantages, it has been found that many peptides do not possess the physicochemical properties necessary to exert the desired biological action, which may lead to certain limitations for their use in the pharmaceutical industry. AMPs are unstable and depend on the environment, the presence of proteases, the variation of $\mathrm{pH}$, temperature, etc. and can quickly become ineffective [9-11]. Other characteristics that reduce their in vivo potential are: (a) their hydrophobicity makes it impossible to transport them inside organisms, (b) they are susceptible to proteolytic enzymes, (c) some of them present cytotoxic potential to eukaryotic cells, as well as 
many side effects which occur due to immune system responses and (d) the cost of large-scale production is increased $[9,10]$. To overcome some of these limitations, considerable efforts have been made to improve the stability of synthetic AMPs and reduce their cytotoxicity against eukaryotic cells, by: substituting unusual amino acids or altering terminal regions $[11,12]$ or by encapsulating them into drug delivery systems such as liposomes or polymeric nanoparticles to facilitate their transport $[13,14]$. Among the possible drug delivery strategies, we focused on an original one using polyion complex (PIC self-assemblies) as nanovectors [15]. PIC self-assemblies form because of the electrostatic attractions between oppositely charged polyions composing them. They are easy to prepare, directly in water and highly tunable with $\mathrm{pH}$ [16], ionic strength [17] and temperature [18]. Double hydrophilic copolymers, formed by a neutral and a charged block, can be than used in order to encapsulate therapeutic agents of opposite charge. In this way, small RNA [19], oligopeptides [20-22] or polypeptides [23], and proteins [24-27] could be successfully inserted in such kind of self-assemblies. We decided to use this self-assembled nanovector in order to protect a cationic AMPs from a body environment. A unique study demonstrated the addedvalue of delivering AMPs using PICs approach in the antimicrobial context [20], but to the best of our knowledge it is the first time that this delivery strategy is proposed to improve antitumor potential of AMPs. Poly(ethylene oxide)-poly(acrylic acid) (PEO-PAA) double hydrophilic block copolymer was chosen because PEO, also known as poly(ethylene glycol) (PEG) is a FDA approved biocompatible hydrophilic block widely used in biology. It forms a hydrophilic shell to protect the inner part of the self-assembly composed by the PAA block, negatively charged and thus able to bind with cationic AMP P6 (HRWWRWWRR-NH $\mathrm{NH}_{2}$. P6 is a peptide previously studied $[6,28]$ which showed also good antimicrobial properties. This 9 amino acids long peptide has a net charge of +5 , which gives it a higher affinity for bacterial and cancer cell membranes and 4 tryptophan residues which ensures a good anchoring into the lipid bilayer. Here we report our results about the formation of an original PICs-peptide delivery system and its efficiency against 3D cell culture model named spheroid.

\section{Materials and Methods}

\subsection{Materials}


P6 was synthetized by ThermoFisher Scientific/Pierce Biotechnology (Rockford, IL, USA) based on the structure reported previously [28]. Poly(ethylene oxide)-poly(acrylic acid), $\mathrm{PEO}_{5 \mathrm{k}-}$ PAA $_{3.2 \mathrm{k}}\left(\mathrm{M}_{\mathrm{n}}=8200\right)$ was purchased from Polymer Source (Montreal, Canada) and used as received. Phosphate buffer saline (PBS) was purchased from Sigma Aldrich (Merck, Darmstadt, Germany). Dimethyl sulfoxide (DMSO) was purchased from Merck (Darmstadt, Germany), 3-(4,5dimethylthiazol-2-yl)-2,5-diphenyltetrazolium bromide (MTT) was purchased from Serva (Heidelberg, Germany). All cell cultivation media and reagents were purchased from Biochrome AG (Berlin, Germany).

\subsection{PICs preparation}

Stock solution of P6 peptide $(4.26 \mathrm{mM})$ was prepared in PBS. The charge ratio, $\mathrm{R}$, is defined as the ratio between the negative charges of the acrylate groups in the copolymer, AA, and the positive charges of the peptide P6 coming from the four arginine residues and the terminal $\mathrm{NH}_{2}$ group as described in equation (1):

$$
\mathrm{R}=\frac{5 \times[\mathrm{P} 6]}{44 \times[\mathrm{PEO}-\mathrm{PAA}]}
$$

In order to prepare the self-assembled PICs of the polymer and the peptide (PEO5k-PAA3.2k: P6), $553 \mu \mathrm{l}$ of the peptide stock solution $(0.00236 \mathrm{mmol}$; [P6]x $5=21.3 \mathrm{mM})$ were added to $2.19 \mathrm{mg}$ of polymer PEO5k-PAA3.2k each containing 44 AA units $(0.000267 \mathrm{mmol}$; [AA] $=44 *$ [PEO-PAA] $=21.25 \mathrm{mM})$. $\mathrm{R}$ is equal 1 in our case as the number of negative charges of the acrylic acid of the polymer are equal to the positive charges of the P6 peptide.

Polymer alone solution $\left(\mathrm{PEO}_{5 \mathrm{k}}-\mathrm{PAA}_{3.2 \mathrm{k}}\right.$ at $\left.0.472 \mathrm{mM}\right)$ and $\mathrm{P} 6$ alone were prepared in PBS as control conditions. These solutions were used within 2 days for physico-chemical characterization experiments.

For in vitro studies we had to dilute the stock solutions as presented in Table 1.

Table 1: Experimental conditions used for in vitro studies

\begin{tabular}{c|c|c} 
Dilutions & PEO-PAA $(\boldsymbol{\mu M})$ & P6 $(\boldsymbol{\mu M})$ \\
\hline D5 & 0.0006 & 6 \\
\hline D4 & 0.0013 & 12 \\
\hline D3 & 0.0025 & 24 \\
\hline D2 & 0.0050 & 48 \\
\hline D1 & 0.010 & 96 \\
\hline
\end{tabular}




\subsection{Transmission Electron Microscopy (TEM)}

The morphology of the nano-objects was visualized by TEM (Hitachi HT7700 microscope, accelerating voltage of $75 \mathrm{kV}$ ). A drop of particle suspensions diluted 1/5 in PBS was deposited onto a discharged copper grid coated with a carbon membrane and wiped with filter paper. The negative staining of the sample by uranyl acetate was conducted for $30 \mathrm{~s}$, and the grid was then dried under a lamp for three minutes. PICs mean diameter was measured on at least 100 nanoobjects on 3 independent pictures.

\subsection{Dynamic Light Scattering (DLS)}

The particle sizes and size distributions of the nano-objects were measured by DLS (Malvern Zetasizer NanoZS $)$ equipped with a He-Ne laser $(\lambda=633 \mathrm{~nm})$ in plastic cuvette on filtered solutions. The correlation function was then analyzed via the general-purpose non-negative least squares (NNLS) method to obtain the intensity-weighted distribution of diffusion coefficients (D) of the solutes. This distribution can be converted, considering Mie theory, to a number-weighted distribution describing the relative proportion of multiple components in the sample based on their number rather than based on their scattering intensity. From the so obtained distribution, the average apparent hydrodynamic diameter, $\mathrm{D}_{\mathrm{h}}$, was determined using the Stokes-Einstein equation. The typical accuracy for these measurements was $10-15 \%$. It has to be mentioned that the determination of $\mathrm{D}_{\mathrm{h}}$ assumed non interacting particles modeled with a homogeneous spherical hard sphere model.

\subsection{Circular dichroism (CD)}

Diluted solutions of PEO-PAA (80-fold), P6 and PICs (240-fold) were used for CD measurements as well UV spectra, with a Jasco J-815 spectropolarimeter. At least $2 \mathrm{~mL}$ of each solution were used in standard quartz cells (P selected cells,10 mm OP). All the measurements were performed at $20^{\circ} \mathrm{C}$, in a 320-200 $\mathrm{nm}$ wavelength range, $1 \mathrm{~nm}$ data pitch, $2 \mathrm{~nm}$ bandwidth, 10 $\mathrm{nm} / \mathrm{min}$ scanning speed, continuous scanning mode, $4 \mathrm{~s}$ DIT and no accumulation.

\subsection{Steady state spectrofluorimetry}


Diluted solutions of PEO-PAA (80-fold), P6 and PICs (240-fold) were used for emission spectra recording thanks to a Horiba Fluorolog 3-2 iHR320 spectrofluorimeter at $20^{\circ} \mathrm{C}$. The excitation wavelength was fixed at $280 \mathrm{~nm}$ and the emission wavelength range was 310-500 $\mathrm{nm}$. The integration time was $100 \mathrm{~ms}, 2 \mathrm{~nm}$ bandwidth (=0.95 mm slitwidth). Excitation (R) and emission (S) signals were corrected considering the technical specifications of the apparatus and a dark offset was applied. Results are given as $S_{\text {corrected }} / R_{\text {corrected }}\left(S_{c} / R_{c}\right)$ for full correction.

\subsection{Cell Culture}

Human colorectal adenocarcinoma HTC-116 cells, recently obtained from ATCC (\#CCL-247) were cultured in Dulbecco's Modified Eagle Medium (DMEM) supplemented with $10 \%$ fetal bovine serum and penicillin-streptomycin $(0.05 \%-100$ units $/ \mathrm{mL})$ in a humidified incubator under an atmosphere containing $5 \% \mathrm{CO}_{2}$. Cells were regularly tested negative for mycoplasma (Lonza $^{\mathrm{TM}}$ Mycoalert ${ }^{\mathrm{TM}}$ Mycoplasma Detection Kit).

\subsection{Spheroid Formation}

The HCT-116 spheroids were formed in a clear, round bottom, ultra-low attachment 96-well microplate (Corning, NY, USA). Briefly, in each well were seeded 5000 cells in a final volume of $200 \mu \mathrm{L}$. After this, the plate was centrifuged for $2 \mathrm{~min}$ and then incubated at $37^{\circ} \mathrm{C}$ for up to 4 days before adding the treatment. Spheroid formation was confirmed by observing the plate under a

light microscope (Olympus CX23 Binocular Microscope, Düsseldorf, Germany). Spheroids were monitored daily and the incubation medium was replaced every 3 days.

\subsection{Treatment of HCT-116 Spheroids}

Treatment evaluation was performed on spheroids in the 4th day after the cells were plated. We applied varying concentrations of P6, PEO-PAA and PICs. The changes in spheroid integrity were evaluated by light microscopy at 24 and $48 \mathrm{~h}$ after treatment.

\subsection{Cell Viability Assays}

Cell viability was assessed using the MTT assay as described previously [29]. After desired treatment times ( 24 and $48 \mathrm{~h}$ ) the culture medium was removed from each well, MTT was added at a final concentration of $1 \mathrm{mg} / \mathrm{mL}$ and the spheroids were further incubated at $37^{\circ} \mathrm{C}$ for $4 \mathrm{~h}$. Next, the medium was removed and DMSO was added to dissolve the formazan crystals formed. Finally, 
the absorbance was recorded at $\lambda=490 \mathrm{~nm}$ using a plate reader (Mithras LB 940, Berthold, Germany). Cell viability was calculated using the following formula:

$\%$ viable cells $=($ Corrected absorbance of treated cells/Corrected absorbance of control cells $) \times$ 100

\subsection{Analysis of Combination Index (CI)}

The interactions between PEO-PAA and P6 were evaluated using the combination index (CI) described by Chou and Talalay, and calculated using Compusyn software. CI $>1$ indicates drug antagonism, $\mathrm{CI}=1$ indicates a drug additive effect, while $\mathrm{CI}<1$ indicates drug synergism.

\subsection{Statistical Analysis}

Each experiment was performed at least three times with at least 3 spheroids per condition, per experiment. All data are presented as means \pm standard deviations, if not stated otherwise. The statistical analysis was carried out using the GraphPad Prism 5 software package (San Diego, CA, USA). One-way and Two-way analysis of variance (ANOVA) were used to calculate statistical significance.

\section{Results and discussions}

3.1. Combination of P6 antimicrobial peptide with PEO-PAA copolymers allowed to form well-defined PIC self-assemblies

The PIC self-assemblies were prepared by simply mixing of PEO-PAA and P6 antimicrobial peptide in PBS at a specific charge ratio of 1 between negatives charges of PAA and positive charges of P6. Transmission electron microscopy analyses (Figure 1A) revealed that P6 alone aggregated into giant tree-like structures, while when associated with copolymers it formed welldefined spherical nano-objects of approximately $21 \mathrm{~nm}$ of diameter. In order to characterize the hydrodynamic size of these nano-objects, DLS measurements were performed on the solutions of the $\mathrm{PEO}_{5 \mathrm{k}}-\mathrm{PAA}_{3.2 \mathrm{k}}$ : P6 (Figure 1B). The solutions of the P6 peptide alone and PEO-PAA polymer alone were also analysed as controls. As expected, PEO-PAA polymer alone solution was characterized by a very low count rate (255 counts/sec), indicative of the absence of selfassemblies. The value of the apparent hydrodynamic diameter, $5 \mathrm{~nm}$, corresponds to free polymer 
chains in solution. The P6 peptide alone solution showed the presence of very large polydisperse $\left(D_{h}=775 \mathrm{~nm}, \mathrm{PDI}=0.4\right)$ aggregates which can also be observed in TEM images (Figure 1A). These big aggregates disappeared when combined with PEO-PAA in PICs, leading to self-assemblies with an apparent hydrodynamic diameter $D_{h}=20 \mathrm{~nm}$. The DLS analysis on a PICs solution diluted 4 times indicated that this high polydispersity index was due to a negligible population produced by aggregation between PICs, (Figure S1 and Table S1).

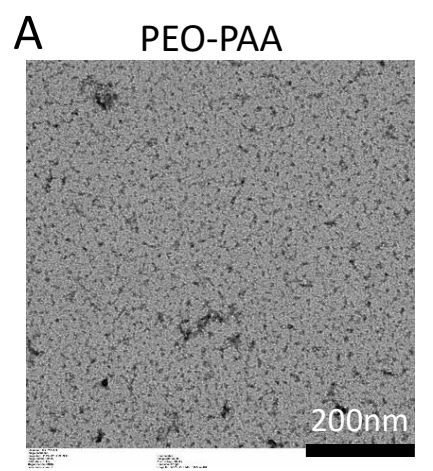

B

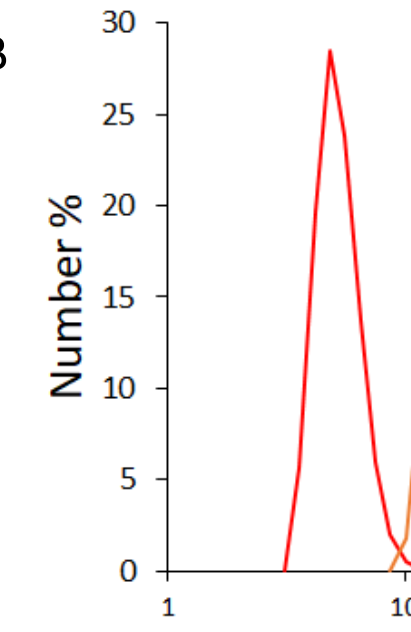

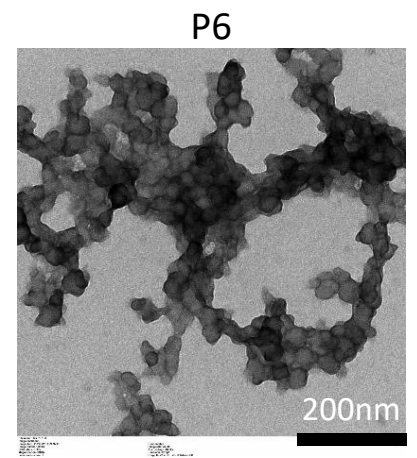

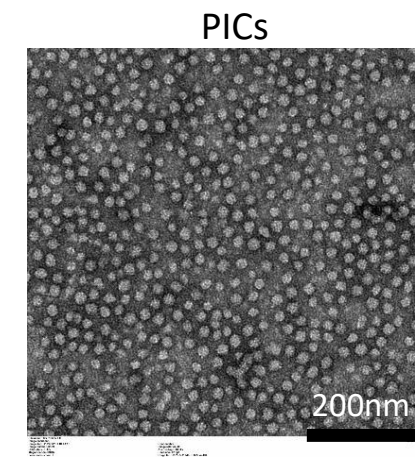

-PEO-PAA

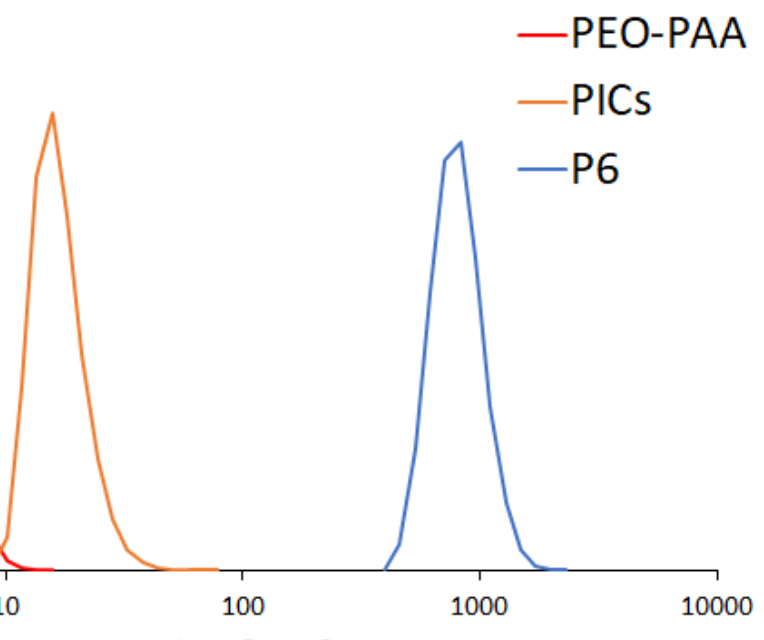

\section{Size $[\mathrm{nm}]$}

Figure 1. Morphological and size distribution features of PEO-PAA alone, P6 alone and PICS micelles. A. Visualization of the different samples by TEM. B. Size distribution of the number weighted hydrodynamic diameter, $D_{h}$, determined by DLS.

It appears that PICs of PEO-PAA charged block copolymers associated with cationic antimicrobial peptide P6 were water-soluble, displaying average diameters around $20 \mathrm{~nm}$ as demonstrated by TEM and DLS analyses (Table 2). The size of these self-assemblies is quite smaller than the one observed in the case of PICs produced with the anionic copolymer methoxy poly(ethylene glycol)- $b$-poly( $\alpha$-glutamic acid), associated with another antimicrobial peptide (MSI-78) (mean diameter of $195.7 \mathrm{~nm}$ ) [20]. In the literature PICs sizes ranging from some tenths 
of nanometers to few hundred of nanometers have been reported [30]. We have previously demonstrated that the size and the internal structure of such self-assemblies depends on the molecular weight of the charged block in the copolymer used [31, 32].

Table 2. DLS and TEM characterization of PEO $\mathrm{O}_{5 k}-P A A_{3.2 k}$ alone, P6 alone and PICs self-assemblies at a charge ratio 1 PEO Sk-PAA $_{3.2 k}$ : P6. PDI: polydispersity index. $D_{h}$ : number weighted hydrodynamic diameter $(\mathrm{nm})$. D: diameter in $\mathrm{nm} \pm$ standard error. na: non-applicable

\begin{tabular}{l|c|c|c} 
& Number weighted $\mathbf{D}_{\mathbf{h}}(\mathbf{D L S})$ & PDI & Average D (TEM) \\
\hline PEO $_{\text {5k-PAA3.2k }}$ & 5 & 0.3 & na \\
\hline P6 & 775 & 0.4 & na \\
\hline PICs & 20 & 0.5 & $20.8 \pm 1.4$ \\
\hline
\end{tabular}

In order to confirm the presence of peptide P6 in the interior of the self-assemblies, both absorption and fluorescence measurements were performed. The spectrophotometry (Figure 2A) results showed that PEO-PAA alone spectrum displays a weak absorption in the interesting wavelength range (200-240 nm) and no absorption from $240 \mathrm{~nm}$ to visible wavelength range. Interestingly, PICs presented quite the same spectrum than P6 alone with the exception of a small red shift $(3 \mathrm{~nm})$ of the $\lambda_{\max }$. That means that the spectrum of the PICs is mostly due to P6 and somehow influenced by the presence of the copolymer. Fluorescence measurements bring to similar conclusions. The emission spectra (Figure 2B) proved the negligible emission of PEOPCL, while PICs emission spectrum showed a blue shift (approx. $7 \mathrm{~nm}$ ) versus the emission of P6. Considering both the red shift of the absorption and the blue shift of the emission when P6 and PEO-PAA are mixed together, it appears clearly a shorter Stokes shift for the fluorescence of P6 when encapsulated within the PICs compared to P6 alone. The smaller the Stokes shift, the closer the conformation between the excited and ground states. That means that P6 is more constrained in PICs than when alone in solution, leading to the conclusion of its interaction with the copolymer chains in the interior of the self-assemblies. Circular dichroism spectra are reported in Figure 2C, and show that the signal is only due to P6. The differences between the spectra of P6 and PICs lead to the conclusion that there is a conformational change for P6 in the self-assemblies, giving another proof that P6 is present in the PIC nano-objects. 


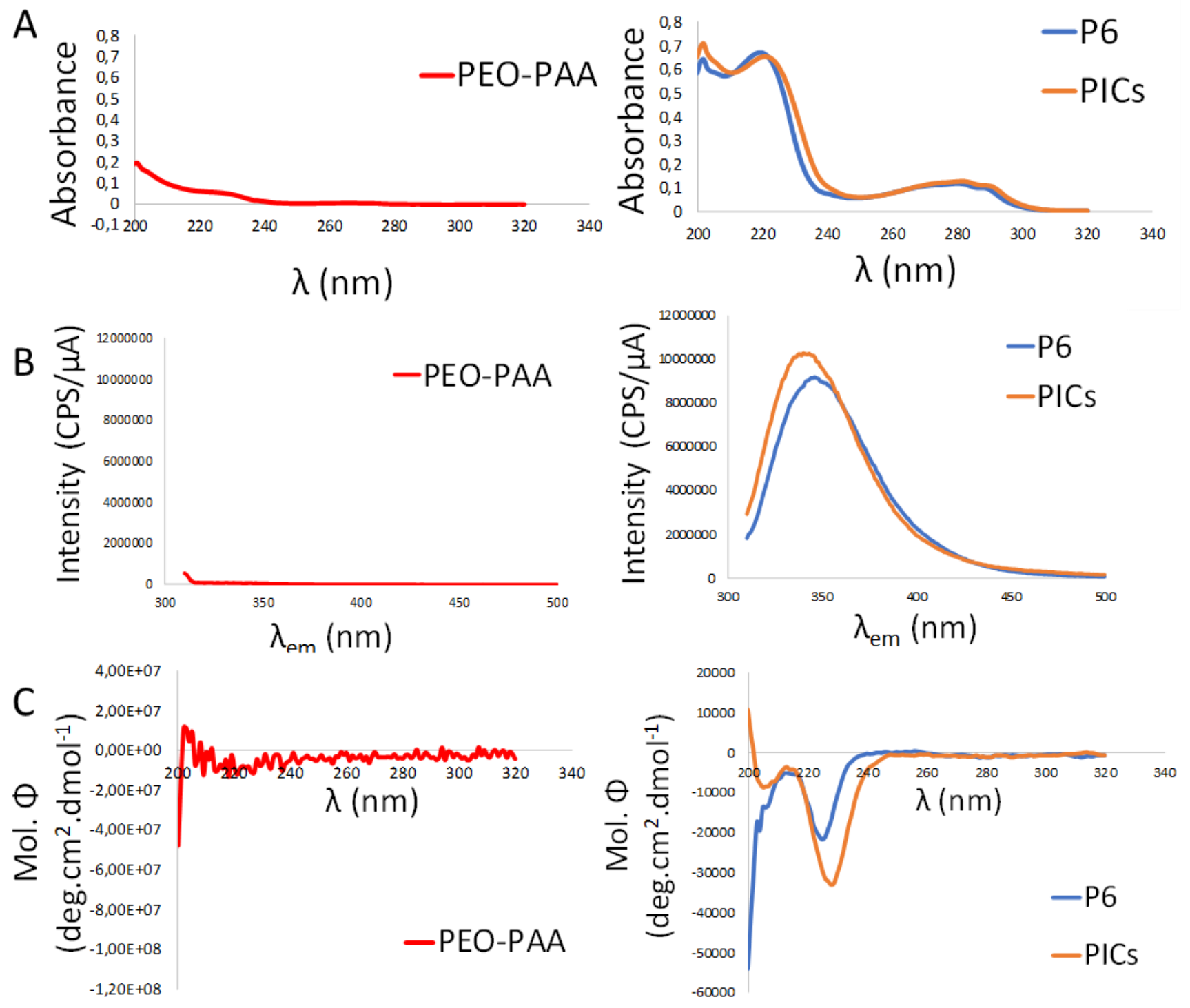

Figure 2: Characterization of PEO-PAA alone, P6 alone and P6 encapsulated in PIC self-

assemblies. A. UV-vis absorption spectra in PBS at $20^{\circ}$ C. B. Emission fluorescence spectra (280nm excitation wavelength) in PBS at $20^{\circ} \mathrm{C}$. C. Circular dichroism spectra (molar ellipticity) in $\mathrm{PBS}$ at $20^{\circ} \mathrm{C}$.

Solutions were diluted for analysis: PEO-PAA (80-fold), P6 and PICs (240-fold).

3.2. PIC self-assemblies, but not polymer or P6 alone, altered macroscopic aspect of HCT116 spheroids

The effect induced by the treatments against the macroscopic morphology of the spheroids was investigated at $24 \mathrm{~h}$ and $48 \mathrm{~h}$ over the entire range of concentrations tested (Figure 3 ). The results 
showed that at the concentrations reported neither the polymer nor the peptide alone (Figure $3 \mathrm{~A}$ and B) alter significantly the area and morphology of the spheroids.

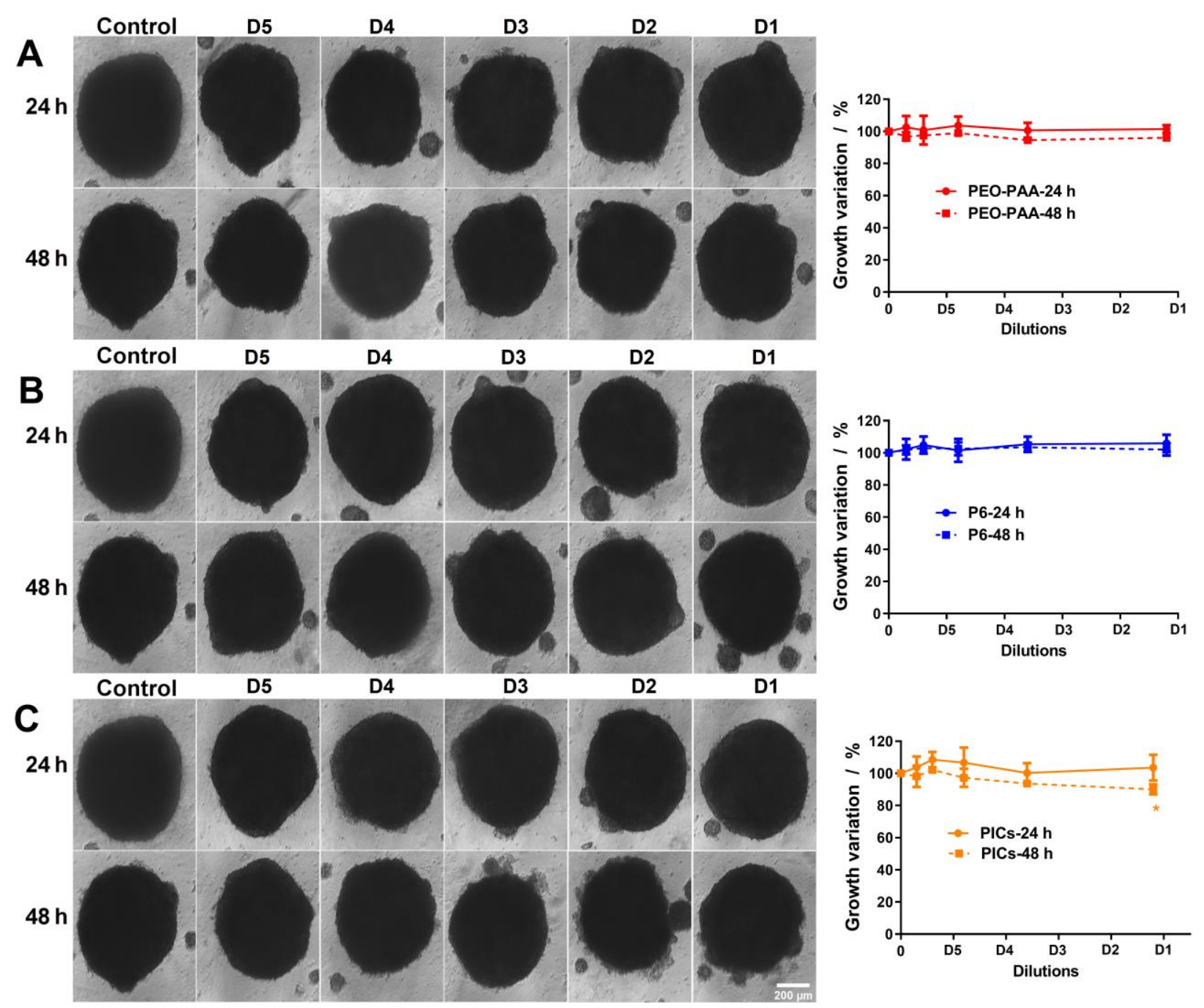

Figure 3. Spheroid microscopy images recorded at 24 and 48 hours after treatment. (A) Spheroids treated with polyethylene oxide - polyacrylic acid polymer alone (PEO-PAA); (B) Spheroids treated with peptide P6 alone, (C) Spheroids treated with the PICs. In the last column are reported the growth variation of treated spheroids $(n=3)$. The images were taken using a $4 x$ microscope.

However, when the PICs were applied, we observed a slight decrease in the size of the spheroids, especially after a $48 \mathrm{~h}$ treatment. For the higher concentration of PICs applied for $48 \mathrm{~h}$ one can also observe that dead cells started to detach from the spheroids (Figure $3 \mathrm{C}$ ) indicating that the treatment should also have an effect on the cell viability of the spheroid. Moreover, P6 and 
PICs penetration in the spheroids was showed using PICs prepared with P6 covalently linked with Rhodamine (Figure S2). For untreated spheroids (Figure S2.A) or spheroids treated with only PEO-PAA (Figure S2.C) we recorded the weak autofluorescence of the spheroids. When the spheroids were treated with P6 (Figure S2.B) or PICs (Figure S2.D) the fluorescence intensity increases. Based on the fluorescence images and the cross-section through the spheroids (Figure S2.E) we showed that P6 and PICs can penetrate the spheroids, especially to its core.

\subsection{PIC self-assemblies increased antitumor potential of P6 against HCT-116 spheroids}

Cell viability of HCT-116 spheroids after treatment is reported in figure 4. For all conditions a constant decrease in cell viability with increasing concentration was observed. This point is interesting because it appears that both components of the PICs present antitumor activity on their own, which is clearly an added-value to improve therapeutic outcomes. For PEO-PAA alone the cell viability was affected significantly starting from $0.0013 \mu \mathrm{M}(\mathrm{D} 4)$ and decreased at the highest concentration $(0.01 \mu \mathrm{M})$ down to $85 \%$. Similar effects were observed when the spheroids were treated for $48 \mathrm{~h}$, with highest concentration reducing the viability down to $80 \%$. Although polymers are used as delivery devices for a large number of molecules and drugs, they can also have a toxic effect against eukaryotic cells on their one. Similar to our results, Turk et al. showed that PAA and various PAA-PEO copolymers show increased toxicity against both normal fibroblast cells and HeLa tumoral cells [33].

P6 alone decreased significantly the cell viability both at $24 \mathrm{~h}$ and $48 \mathrm{~h}$. After $24 \mathrm{~h}$ the highest concentration of P6 reduced the cell viability to $80 \%$, while after $48 \mathrm{~h}$ the viability was only $75 \%$. We showed previously that the antimicrobial peptide P6 has a low toxicity against normal skin cells up to $200 \mu \mathrm{M}$ [28], a concentration higher than the one we found to show effect against HCT116 spheroids. Similar to our results, there were numerous tryptophan rich AMPs applied efficiently against cancer cells grown in monolayer [34-37]. However, we reported previously the effect of Gramicidn A against HT-29 spheroids showing that the peptide alone is efficient, destabilizing the spheroids, while in combination with a tumoral drug the effect is amplified [29].

Finally, for the PIC nano-objects loaded with the antimicrobial peptide P6 a more pronounced decrease can be observed both at 24 and 48 h. For HCT-116 spheroids treated with PICs for $24 \mathrm{~h}$ 
the cell viability decreases down to $65 \%$ at the highest concentration. Only at the highest concentration we have a significant difference in cell viability as compared with PEO-PAA or P6 alone. However, when spheroids were treated for $48 \mathrm{~h}$ with PICs, the results obtained for concentrations starting $12 \mu \mathrm{M}$ (D4) showed a significant decreased both compared to PEO-PAA and P6 alone, as well as the treatment applied for $24 \mathrm{~h}$. Thus, at the highest concentration tested, the cells viability was down to $56 \%$. To our knowledge this is the first time reporting the effect induced by a peptide encapsulated into a polyion complex against cancer spheroids.
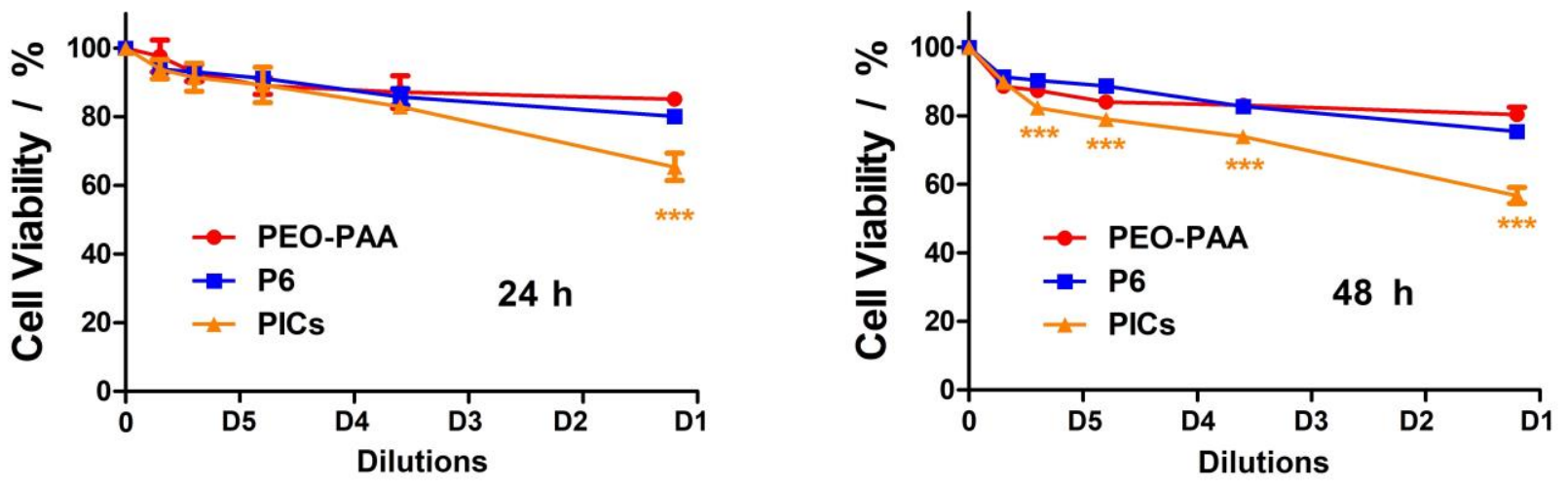

Figure 4. Cell viability of HCT-116 spheroids treated with increasing concentrations of PEO-PAA, P6 and PICs (at 24h and 48h). One-way ANOVA analysis with Dunnett's Multiple Comparison post-test shows a significant difference between treated spheroids and control. Two-way ANOVA analysis shows a significant difference between the highest concentrations of PEO-PAA vs PICs and P6 vs PICs $p<0.001$ at $24 \mathrm{~h}$ ) and for all concentrations of PEO-PAA vs PICs and P6 vs PICs ( $p<0.001$ at $48 \mathrm{~h})$.

Finally, we checked whether the combination of the two compounds PEO-PAA and P6 within the PIC self-assemblies has a synergistic, additive of antagonism effect on its antitumor properties. For that purpose, the combination index $(\mathrm{CI})$ and the fraction affected $(\mathrm{Fa})$ were calculated and reported in Figure 5A and B, and the CI as a function of the fraction affected $(\mathrm{Fa})$ was plotted for all PICs tested combinations (Figure 5C). The combination index is obtained from the doseresponse data and can give a quantitative measure of the effects produced by drug combination, whereas the fraction affected denotes the effect induced by the drug tested. Based on Chou paper [38] a CI values $<1$ is indicative of a synergistic effect, $\mathrm{CI}=1$ for additivity, whereas $\mathrm{CI}>1$ indicates antagonism. A synergistic effect was found for one condition when spheroids were treated for 24 $\mathrm{h}$ and almost all the concentrations when treated for $48 \mathrm{~h}$. Interestingly, the longer the incubation 
was, the more the synergistic effect of the association of P6 with the polymer in PIC selfassemblies was clear.
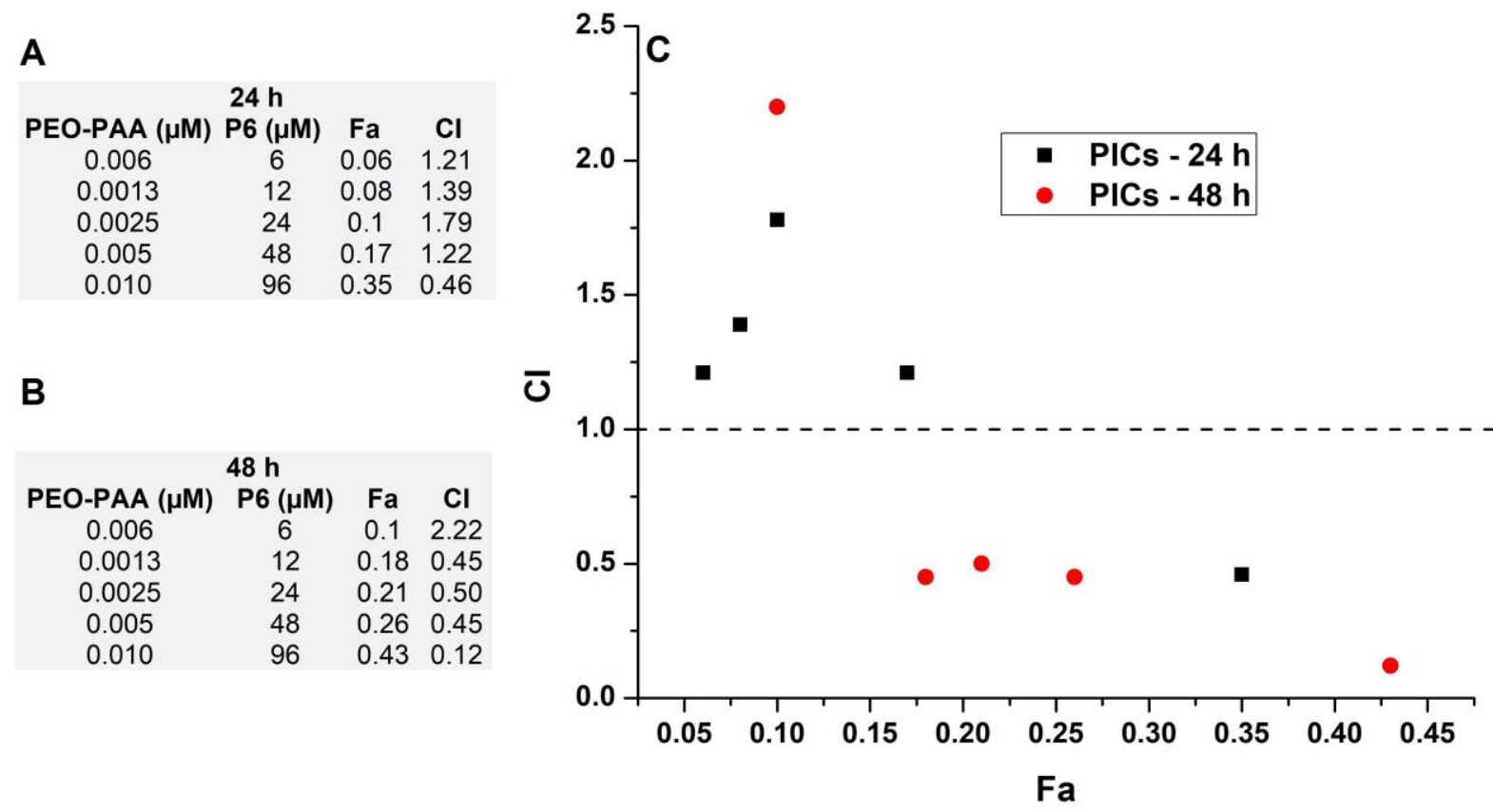

Figure 5. Effects of combination therapy of PEO-PAA and P6 on HCT-116 spheroids. Combination index (CI) and fraction affected (Fa) values at the concentrations tested for $24 h(A)$ and $48 h(B)$. (C) CI versus Fa values plotted for all the experimental conditions tested for PICs at 24 and $48 \mathrm{~h}$. CI<1 indicates synergism, $C I=1$ additivity, whereas $C I>1$ indicates antagonism.

Observing the synergistic antitumor effects of PICs which combine the antimicrobial peptide with poly(ethylene oxide)-poly(acrylic acid) (PEO-PAA) copolymer, we conclude that understanding how to optimize the preparation of these particles and their stability is of key importance for the development of biomedical applications of this type of nanomaterials. Applications of these types of polyion complexes nano-objects are reported in literature for transport of genes, proteins and small molecules such as various therapeutic compounds [39]. These PIC delivery systems encapsulating AMPs can be used on several types of cell lines, but also in combination with classical treatment schemes in order to see the possible synergistic effects of the peptides. With the implementation of this delivery system, a study of the mechanism of action of peptides on tumor cells is required, especially looking at the cell death pathways involved. Tracking protein expression, but also genetic, is imperative to prove the effectiveness of 
this type of treatment which aims to reduce and even eliminate the side effects and injuries caused by antitumor treatments currently used.

\section{Conclusions}

This study showed the formation of a polyion complex in combination with an antimicrobial peptide used for the first time for antitumor purpose at tissue scale. PIC self-assemblies thanks to the electrostatic interaction between the cationic AMP P6 and the anionic block copolymer poly(ethylene oxide)-poly(acrylic acid) (PEO-PAA) were successfully prepared and characterized. It proved its antitumor potential onto human 3D tumor spheroid model by significantly decreasing cell viability in a synergistic manner.

\section{Declaration of Competing Interest}

There are no conflicts of interest to declare.

\section{Acknowledgements}

This research was performed in the scope of PHC Brancusi 2019 (grant number 43454XF). This work was supported by the Romanian Ministry of Research, Innovation and Digitalization, through the National Core Program No. PN 190602 03/2019; and a grant CCCDI-UEFISCDI, project numbers PN-III-P3-3.1-PM-RO-FR-2019-0147/12BM/2019. We would like to thanks AnneFrançoise Mingotaud for fruitful scientific discussions on PICs physico-chemical characterization and Clément Roux for its help with the design of the graphical abstract.

\section{References}

1. Siegel, R.L., Miller, K.D., and Jemal, A.; Cancer statistics, 2020, CA: Cancer J. Clin., 2020, 70, 7-30, doi: 10.3322/caac. 21590 .

2. Choi, D.K., Helenowski, I., and Hijiya, N.; Secondary malignancies in pediatric cancer survivors: perspectives and review of the literature, Int. J. Cancer, 2014, 135, 1764-73, doi: 10.1002/ijc.28991. 
3. Cederlund, A., Gudmundsson, G.H., and Agerberth, B.; Antimicrobial peptides important in innate immunity, FEBS J., 2011, 278, 3942-51, doi: 10.1111/j.1742-4658.2011.08302.x.

4. Hancock, R.E. and Diamond, G.; The role of cationic antimicrobial peptides in innate host defences, Trends Microbiol., 2000, 8, 402-10, doi: 10.1016/s0966-842x(00)01823-0.

5. Brogden, K.A.; Antimicrobial peptides: pore formers or metabolic inhibitors in bacteria?, Nat. Rev. Microbiol., 2005, 3, 238-50, doi: 10.1038/nrmicro1098.

6. Bacalum, M., Janosi, L., Zorila, F., Tepes, A.M., Ionescu, C., Bogdan, E., Hadade, N., Craciun, L., Grosu, I., Turcu, I., and Radu, M.; Modulating short tryptophan- and argininerich peptides activity by substitution with histidine, Biochim. Biophys. Acta, Gen. Subj., 2017, 1861, 1844-1854, doi: 10.1016/j.bbagen.2017.03.024.

7. Ward, P.P., Paz, E., and Conneely, O.M.; Multifunctional roles of lactoferrin: a critical overview, Cell. Mol. Life Sci., 2005, 62, 2540-8, doi: 10.1007/s00018-005-5369-8.

8. Riedl, S., Zweytick, D., and Lohner, K.; Membrane-active host defense peptides-challenges and perspectives for the development of novel anticancer drugs, Chem. Phys. Lipids, 2011, 164, 766-81, doi: 10.1016/j.chemphyslip.2011.09.004.

9. Lee, I.H., Cho, Y., and Lehrer, R.I.; Effects of $\mathrm{pH}$ and salinity on the antimicrobial properties of clavanins, Infect. Immun., 1997, 65, 2898-903, doi: 10.1128/iai.65.7.28982903.1997.

10. Rozek, A., Powers, J.P., Friedrich, C.L., and Hancock, R.E.; Structure-based design of an indolicidin peptide analogue with increased protease stability, Biochemistry, 2003, 42, 14130-8, doi: 10.1021/bi035643g.

11. John, H., Maronde, E., Forssmann, W.G., Meyer, M., and Adermann, K.; N-terminal acetylation protects glucagon-like peptide GLP-1-(7-34)-amide from DPP-IV-mediated degradation retaining cAMP- and insulin-releasing capacity, Eur. J. Med. Res., 2008, 13, 73-8, doi:

12. McPhee, J.B., Scott, M.G., and Hancock, R.E.; Design of host defence peptides for antimicrobial and immunity enhancing activities, Comb. Chem. Hiigh Throughput Screen., 2005, 8, 257-72, doi: 10.2174/1386207053764558.

13. Wang, J., Hu, X., and Xiang, D.; Nanoparticle drug delivery systems: an excellent carrier for tumor peptide vaccines, Drug Deliv., 2018, 25, 1319-1327, doi: 10.1080/10717544.2018.1477857.

14. Kovalainen, M., Monkare, J., Riikonen, J., Pesonen, U., Vlasova, M., Salonen, J., Lehto, V.P., Jarvinen, K., and Herzig, K.H.; Novel delivery systems for improving the clinical use of peptides, Pharmacol. Rev., 2015, 67, 541-61, doi: 10.1124/pr.113.008367.

15. Kataoka, K., Harada, A., and Nagasaki, Y.; Block copolymer micelles for drug delivery: design, characterization and biological significance, Adv. Drug Deliv. Rev., 2001, 47, 113 31, doi: 10.1016/s0169-409x(00)00124-1.

16. Cingil, H.E., Meertens, N.C.H., and Voets, I.K.; Temporally Programmed Disassembly and Reassembly of C3Ms, Small, 2018, 14, e1802089, doi: 10.1002/smll.201802089. 
17. Lindhoud, S., Voorhaar, L., de Vries, R., Schweins, R., Cohen Stuart, M.A., and Norde, W.; Salt-induced disintegration of lysozyme-containing polyelectrolyte complex micelles, Langmuir, 2009, 25, 11425-30, doi: 10.1021/la901591p.

18. Read, E., Lonetti, B., Gineste, S., Sutton, A.T., Di Cola, E., Castignolles, P., Gaborieau, M., Mingotaud, A.F., Destarac, M., and Marty, J.D.; Mechanistic insights into the formation of polyion complex aggregates from cationic thermoresponsive diblock copolymers, J. Colloid Interface Sci., 2021, 590, 268-276, doi: 10.1016/j.jcis.2021.01.028.

19. Koji, K., Yoshinaga, N., Mochida, Y., Hong, T., Miyazaki, T., Kataoka, K., Osada, K., Cabral, H., and Uchida, S.; Bundling of mRNA strands inside polyion complexes improves mRNA delivery efficiency in vitro and in vivo, Biomaterials, 2020, 261, 120332, doi: 10.1016/j.biomaterials.2020.120332.

20. Wang, C., Feng, S., Qie, J., Wei, X., Yan, H., and Liu, K.; Polyion complexes of a cationic antimicrobial peptide as a potential systemically administered antibiotic, Int. J. Pharm., 2019, 554, 284-291, doi: 10.1016/j.ijpharm.2018.11.029.

21. Fujita, S., Motoda, Y., Kigawa, T., Tsuchiya, K., and Numata, K.; Peptide-Based Polyion Complex Vesicles That Deliver Enzymes into Intact Plants To Provide Antibiotic Resistance without Genetic Modification, Biomacromolecules, 2021, 22, 1080-1090, doi: 10.1021/acs.biomac.0c01380.

22. Raveendran, R., Chen, F., Kent, B., and Stenzel, M.H.; Estrone-Decorated Polyion Complex Micelles for Targeted Melittin Delivery to Hormone-Responsive Breast Cancer Cells, Biomacromolecules, 2020, 21, 1222-1233, doi: 10.1021/acs.biomac.9b01681.

23. Nita, L.E., Chiriac, A.P., and Bercea, M.; Effect of $\mathrm{pH}$ and temperature upon selfassembling process between poly(aspartic acid) and Pluronic F127, Colloids Surf B Biointerfaces, 2014, 119, 47-54, doi: 10.1016/j.colsurfb.2014.04.023.

24. Gao, G.H., Park, M.J., Li, Y., Im, G.H., Kim, J.H., Kim, H.N., Lee, J.W., Jeon, P., Bang, O.Y., Lee, J.H., and Lee, D.S.; The use of $\mathrm{pH}$-sensitive positively charged polymeric micelles for protein delivery, Biomaterials, 2012, 33, 9157-64, doi: 10.1016/j.biomaterials.2012.09.016.

25. Tao, A., Huang, G.L., Igarashi, K., Hong, T., Liao, S., Stellacci, F., Matsumoto, Y., Yamasoba, T., Kataoka, K., and Cabral, H.; Polymeric Micelles Loading Proteins through Concurrent Ion Complexation and pH-Cleavable Covalent Bonding for In Vivo Delivery, Macromol. Biosci., 2020, 20, e1900161, doi: 10.1002/mabi.201900161.

26. Qin, X., Yu, C., Wei, J., Li, L., Zhang, C., Wu, Q., Liu, J., Yao, S.Q., and Huang, W.; Rational Design of Nanocarriers for Intracellular Protein Delivery, Adv. Mater., 2019, 31, e1902791, doi: 10.1002/adma.201902791.

27. Lee, Y., Ishii, T., Cabral, H., Kim, H.J., Seo, J.H., Nishiyama, N., Oshima, H., Osada, K., and Kataoka, K.; Charge-conversional polyionic complex micelles-efficient nanocarriers for protein delivery into cytoplasm, Angew. Chem. Int. Ed. Engl., 2009, 48, 5309-12, doi: 10.1002/anie.200900064. 
28. Bacalum, M., Dragulescu, E.C., Necula, G., Codita, I., and Radu, M.; Short tryptophanand arginine-rich peptide shows efficacy against clinical methicillin-resistant Staphylococcus aureus strains isolated from skin and soft tissue infections, Sci. Rep., 2019, 9, 17176, doi: 10.1038/s41598-019-53926-4.

29. Raileanu, M., Popescu, A., and Bacalum, M.; Antimicrobial Peptides as New Combination Agents in Cancer Therapeutics: A Promising Protocol against HT-29 Tumoral Spheroids, Int. J. Mol. Sci, 2020, 21, doi: 10.3390/ijms21186964.

30. Sproncken, C.C.M., Magana, J.R., and Voets, I.K.; 100th Anniversary of Macromolecular Science Viewpoint: Attractive Soft Matter: Association Kinetics, Dynamics, and Pathway Complexity in Electrostatically Coassembled Micelles, ACS Macro Lett., 2021, 10, 167179, doi: 10.1021/acsmacrolett.0c00787.

31. Till, U., Gaucher, M., Amouroux, B., Gineste, S., Lonetti, B., Marty, J.D., Mingotaud, C., Bria, C.R.M., Williams, S.K.R., Violleau, F., and Mingotaud, A.F.; Frit inlet field-flow fractionation techniques for the characterization of polyion complex self-assemblies, J. Chromatogr. A, 2017, 1481, 101-110, doi: 10.1016/j.chroma.2016.12.050.

32. Gineste, S., Di Cola, E., Amouroux, B., Till, U., Marty, J.D., Mingotaud, A.F., Mingotaud, C., Violleau, F., Berti, U., Parigi, U., Luchinat, U., Balor, S., Marty, M., and Lonetti, B.; Mechanistic Insights into Polyion Complex Associations, Macromolecules, 218, 51, 14271440, doi: 10.1021/acs.macromol.7b02391.

33. Turk, M., Rzayev, Z.M., and Khalilova, S.A.; Bioengineering functional copolymers. XIV. Synthesis and interaction of poly(N-isopropylacrylamide-co-3,4-dihydro-2H-pyran-altmaleic anhydride)s with SCLC cancer cells, Bioorg. Med. Chem., 2010, 18, 7975-84, doi: 10.1016/j.bmc.2010.09.031.

34. Chu, H.L., Yip, B.S., Chen, K.H., Yu, H.Y., Chih, Y.H., Cheng, H.T., Chou, Y.T., and Cheng, J.W.; Novel antimicrobial peptides with high anticancer activity and selectivity, PLoS One, 2015, 10, e0126390, doi: 10.1371/journal.pone.0126390.

35. Maraming, P., Klaynongsruang, S., Boonsiri, P., Peng, S.F., Daduang, S., Rungsa, P., Tavichakorntrakool, R., Chung, J.G., and Daduang, J.; Anti-metastatic Effects of Cationic KT2 Peptide (a Lysine/Tryptophan-rich Peptide) on Human Melanoma A375.S2 Cells, In Vivo, 2021, 35, 215-227, doi: 10.21873/invivo.12250.

36. Duong, D.T., Singh, S., Bagheri, M., Verma, N.K., Schmidtchen, A., and Malmsten, M.; Pronounced peptide selectivity for melanoma through tryptophan end-tagging, Sci. Rep., 2016, 6, 24952, doi: 10.1038/srep24952.

37. Feng, X., Jin, S., Wang, M., Pang, Q., Liu, C., Liu, R., Wang, Y., Yang, H., Liu, F., and Liu, Y.; The Critical Role of Tryptophan in the Antimicrobial Activity and Cell Toxicity of the Duck Antimicrobial Peptide DCATH, Front. Microbiol., 2020, 11, 1146, doi: 10.3389/fmicb.2020.01146.

38. Chou, T.C.; Drug combination studies and their synergy quantification using the ChouTalalay method, Cancer Res., 2010, 70, 440-6, doi: 10.1158/0008-5472.CAN-09-1947. 
39. Cabral, H. and Kataoka, K.; Progress of drug-loaded polymeric micelles into clinical studies, J. Control Release, 2014, 190, 465-76, doi: 10.1016/j.jconrel.2014.06.042. 